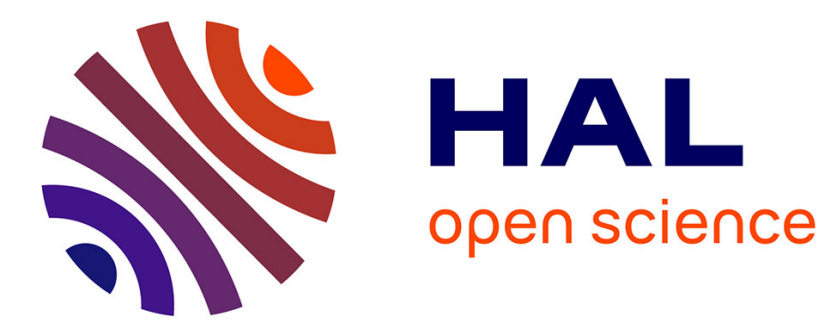

\title{
Study and Optimization of a Non-Foster Circuit for the Design of Wideband Metasurfaces
}

Christophe Fisne, Cédric Martel, Anne-Laure Franc, Nathalie Raveu

\section{To cite this version:}

Christophe Fisne, Cédric Martel, Anne-Laure Franc, Nathalie Raveu. Study and Optimization of a Non-Foster Circuit for the Design of Wideband Metasurfaces. International Conference on Electromagnetics in Advanced Applications (ICEAA'19), Sep 2019, GRENADE, Spain. hal-02351699

\section{HAL Id: hal-02351699 \\ https://hal.science/hal-02351699}

Submitted on 6 Nov 2019

HAL is a multi-disciplinary open access archive for the deposit and dissemination of scientific research documents, whether they are published or not. The documents may come from teaching and research institutions in France or abroad, or from public or private research centers.
L'archive ouverte pluridisciplinaire HAL, est destinée au dépôt et à la diffusion de documents scientifiques de niveau recherche, publiés ou non, émanant des établissements d'enseignement et de recherche français ou étrangers, des laboratoires publics ou privés. 


\section{Study and Optimization of a Non-Foster Circuit for the Design of Wideband Metasurfaces}

\author{
Christophe Fisné \\ ONERA/DEMR \\ Université de Toulouse \\ France \\ christophe.fisne@onera.fr
}

\author{
Cédric Martel \\ ONERA/DEMR \\ Université de Toulouse \\ France \\ cedric.martel@onera.fr
}

\author{
Anne-Laure Franc \\ LAPLACE \\ CNRS, INPT, UPS \\ Toulouse, France \\ franc@laplace.univ-tlse.fr
}

\author{
Nathalie Raveu \\ LAPLACE \\ CNRS, INPT, UPS \\ Toulouse, France \\ raveu@laplace.univ-tlse.fr
}

\begin{abstract}
In this paper, a detailed study and the optimization of a Negative Impedance Converter (NIC) circuit are presented to increase the bandwidth of a passive metasurface. The design and optimization procedure take into account the biasing and the effect of the transistors. The optimization ensures that the load can be realized with passive components.
\end{abstract}

Keywords - active metasurface, active circuit, Non-Foster circuit, NIC

\section{INTRODUCTION}

Metasurfaces represent an important field of research as they can exhibit various interesting characteristics, such as high impedance and perfectly magnetic surfaces, used to improve the antenna performances. It is well-known, however, that metasurfaces suffer from poor bandwidth performance due to their resonant characteristics [1]. One practical way improving the bandwidth of these structures is to use Non-Foster circuits that synthetize negative impedances [2]. The non-Foster characteristic of a circuit is usually obtained with an active circuit commonly called a Negative Impedance Converter (NIC) [3]. In the literature, various NIC designs have been presented. In most cases, however, the designs have been proposed considering that a simple equivalent model of the resonant structure is known. The NIC circuits are then designed to synthetize either a negative inductance and/or a negative capacitance that compensate the reactance of the structure [2], [4]. However, when the equivalent model of the resonant structure cannot be easily determined due to complex geometries, a different approach must be proposed for the design of the non-Foster circuit. This paper proposes a way to overcome this issue. The study is carried out in the $[0.5 \mathrm{GHz} ; 1.5 \mathrm{GHz}]$ frequency band.

First, the general approach for the optimization of the NIC is presented. Then, the characterization of a passive metasurface structure is recalled [5]. Finally, the NIC and its load are designed and optimized by taking into account the biasing and the effect of the transistors. The optimization ensures that the load can be realized with passive components.

\section{GENERAL APPROACH}

The active metasurface is schematized in Fig. 1. It is made of the passive metasurface structure (META), and the NIC circuit and its load. The proposed methodology for designing the wideband metasurface relies on the optimization of the NIC and its load, which are connected to this fixed metasurface resonant structure in order to achieve a reflection coefficient (referred as $\Gamma$ ) strictly equal to one.

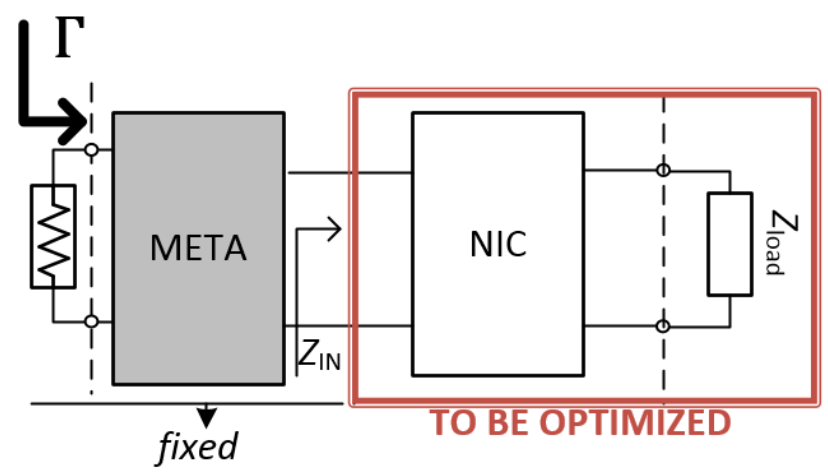

Fig. 1. Active wideband metasurface structure.

\section{CharaCterization OF THE Metasurface}

The metasurface used in this study is shown in Fig. 2. Two Neltec NY9220 $\left(\varepsilon_{\mathrm{r}}=2.2\right.$, $\left.\tan \delta=0.0009\right)$ substrates are stacked above a metallic ground plane. The thicknesses of lower and upper dielectric substrates are respectively 2.286 $\mathrm{mm}$ and $0.127 \mathrm{~mm}$. The periodicity of the unit cell is $24 \mathrm{~mm}$ $\times 24 \mathrm{~mm}$. The $0.127 \mathrm{~mm}$ thick dielectric substrate has square copper patches printed on both sides. The patches of the top layer of the elementary periodic cell are connected 2 by 2 with metallic junctions. The patches of the bottom copper layer are translated with respect to the top copper layer by half the periodicity and do not have any junctions between them. A complete study of the electromagnetic behavior of this structure has been carried out in [5].

Moreover, metalized vias located at the patch junctions are used to connect the top layer to an electronic port placed below the ground plane of the metasurface. Their purpose is to transfer a maximum field to a localized back port when the metasurface is illuminated by a normally incident plane wave. The electronic port is used to plug the NIC circuit to the metasurface.

A full-wave analysis of the metasurface periodic unit cell is carried out using the commercial software HFSS ${ }^{\mathrm{TM}}$. The structure is characterized with two ports: one port above the structure for the plane wave excitation of the reflector and a second port underneath the ground plane for the NIC connection. From this analysis, the S-parameters $(2 \times 2$ matrix) of the metasurface are determined. 


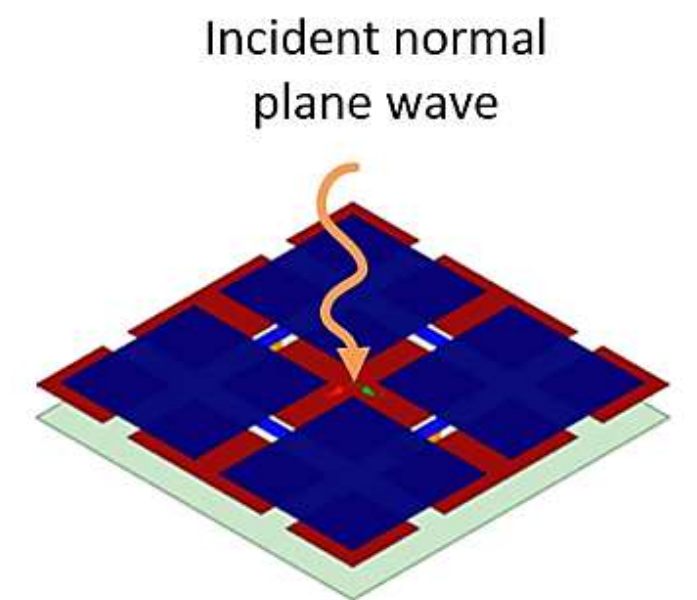

(a) Top view

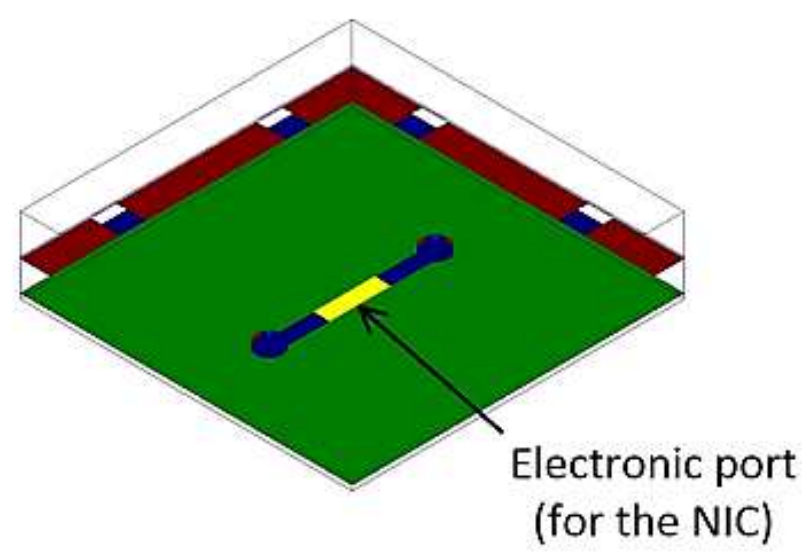

(b) Bottom view

Fig. 2. Elementary cell of the metasurface (dielectric substrates not represented).

By enforcing the $\Gamma$ reflection coefficient of the active metasurface to one, the impedance $Z_{\mathrm{IN}}$ can be computed. The result is plotted in Fig. 3 .

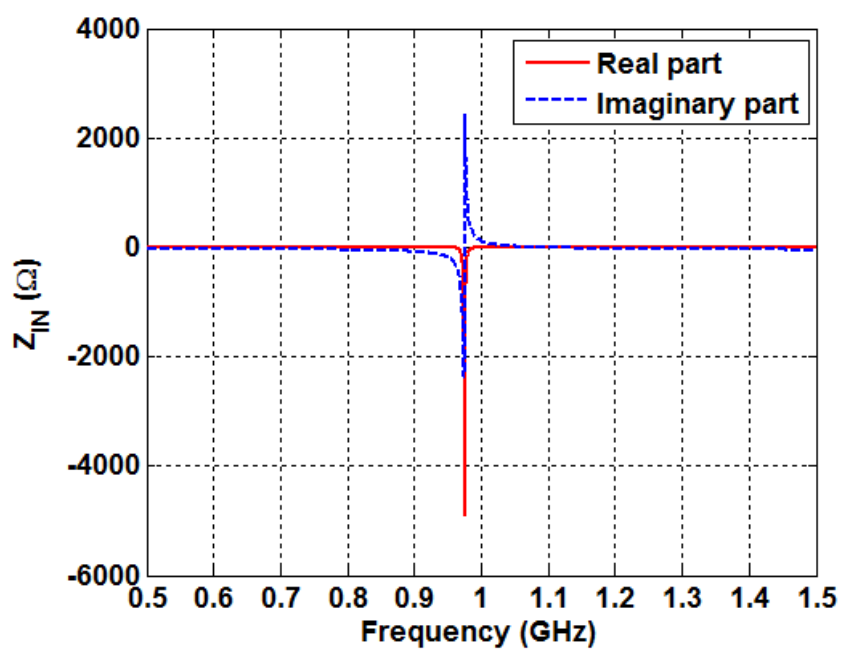

Fig. 3. Metasurface's load impedance to obtain $\Gamma=1$.

The $Z_{\mathrm{IN}}$ impedance is a non-Foster impedance as represented in Fig. 3: The reactive part decreases when the frequency increases, and at the resonance, the real part is both maximum and negative. This result confirms the fact that a NIC circuit is needed to achieve the required $Z_{\mathrm{IN}}$ impedance.

\section{NeGATIVE IMPEDANCE CONVERTER DESIGN}

\section{A. Characteristics of the NIC circuit without biasing}

The NIC circuit used in this study is shown in Fig. 4. It is based on the work presented in [6]. This circuit has been chosen for its simplicity and since no crossover occurs in the RF path. This circuit uses two T1 and T2 bipolar junction transistors, and two $R_{1}$ and $R_{2}$ resistors, which are used to control the reversal factor between the input impedance $Z_{\text {in }}$ at port 1 and the load impedance, $Z_{\mathrm{load}}$ at port 2 [7].

$$
Z_{\text {in }}=-\frac{R_{1}}{R_{2}} Z_{\text {load }}
$$

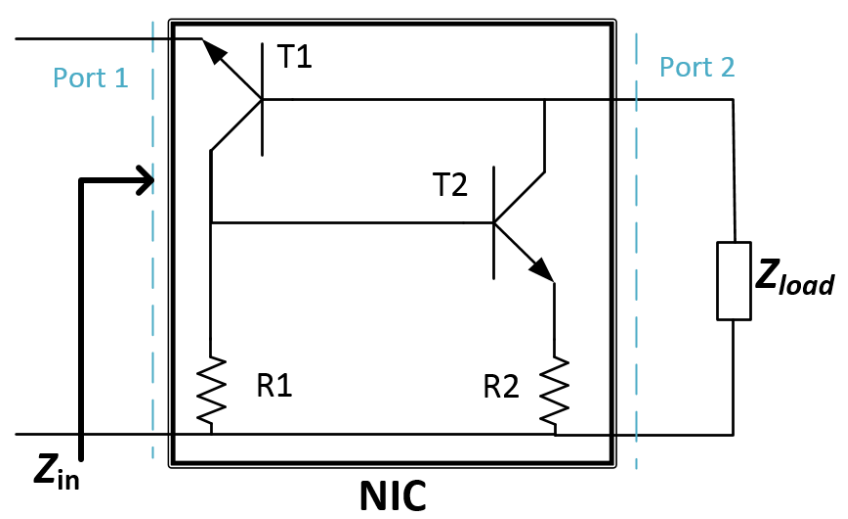

Fig. 4. NIC circuit design [6].

In this study, the BFP720 transistors from Infineon ${ }^{\mathrm{TM}}$ are chosen for their high transition frequency $(14 \mathrm{GHz})$ and good performances especially regarding the impedance reversal linearity in comparison with the other transistors models of the manufacturer.

\section{B. Characteristics of the NIC circuit with biasing}

The use of transistors requires the implementation of a biasing circuit. Fig. 5 shows the DC biasing for a class A power amplifier setup in the NIC circuit.

A special care is given to obtain a biasing independent from $R_{1}$ and $R_{2}$ values using choke coils $\left(L_{\mathrm{S}}=100 \mathrm{nH}\right)$, coupling capacitors $\left(C_{\mathrm{L}}=100 \mathrm{pF}\right)$ and decoupling capacitors $\left(C_{\mathrm{D}}=10 \mathrm{pF}\right)$. The class $\mathrm{A}$ power amplifier biasing is reached with $\mathrm{V}_{\mathrm{CE}}=2 \mathrm{~V}$ and $\mathrm{I}_{\mathrm{C}}=12.5 \mathrm{~mA}$ in both BFP720, which leads to $R_{\mathrm{E} 1}=R_{\mathrm{C} 2}=680 \Omega$ and $R_{\mathrm{B} 1}=R_{\mathrm{B} 2}=22 \mathrm{k} \Omega$.

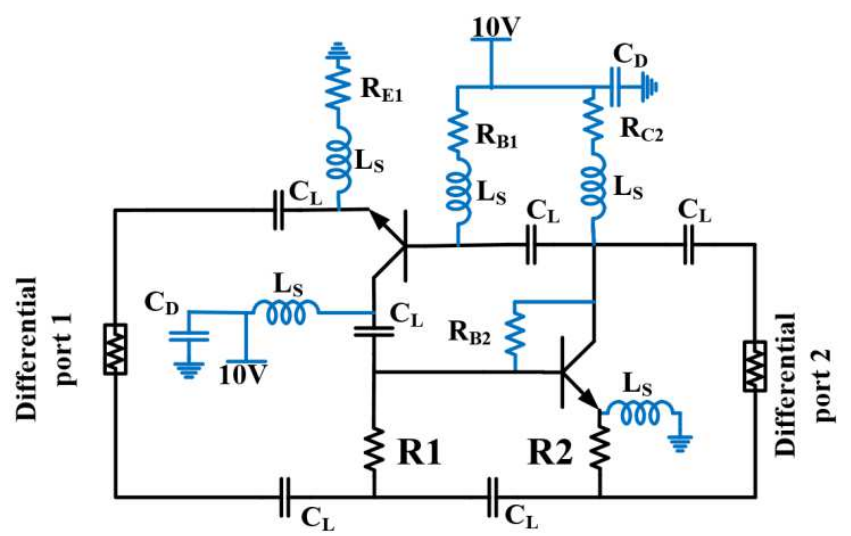

Fig. 5. Circuit design with focus on the DC biasing (illustrated in blue). 
The NIC circuit of Fig. 5 is tested using the circuit simulation software $\mathrm{ADS}^{\mathrm{TM}}$ for a $50 \Omega$-load and $R_{1}$ and $R_{2}$ both equal to $10 \Omega$. The $Z_{\text {in }}$ result is represented in Fig. 6 . It can be observed that the $Z_{\text {in }}$ obtained impedance is not strictly equal to the expected negative value of $-50 \Omega$. Therefore, the equation (1) is partially accurate, and should only be used to outline a trend. The discrepancy between the obtained result and the theory (equation (1)) can be explained by the presence of imperfections in the transistors (especially PN junction capacitors) and the biasing.

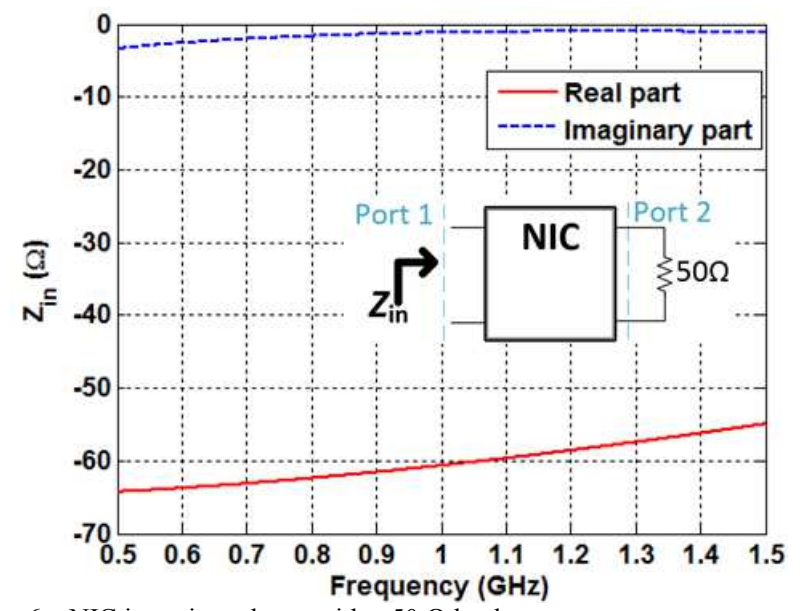

Fig. 6. NIC input impedance with a $50 \Omega$ load.

A more realistic way to calculate the relationship between the load and the input impedance is to consider the $Z_{\mathrm{ij}}$ parameters of the NIC. Hence, equation (2) accurately determinates the load impedance $Z_{\text {load }}$ to connect at port 2 as a function of the input impedance $Z_{\text {in }}$.

$$
Z_{\text {load }}=-Z_{22}-\frac{Z_{12} \cdot Z_{21}}{Z_{\text {in }}-Z_{11}}
$$

Fig. 7 shows the $Z_{\text {load }}$ impedance calculated when the $Z_{\text {in }}$ input impedance is the impedance given in Fig. 3. The $Z_{\text {load }}$ impedance is of Foster type and seems to be a parallel-RLC circuit impedance with a resonant effect observed at $980 \mathrm{MHz}$. However, the real part is slightly negative in the band of interest except at the resonance. This negative real part is not achievable with passive components. Therefore, the NIC circuit must be modified.

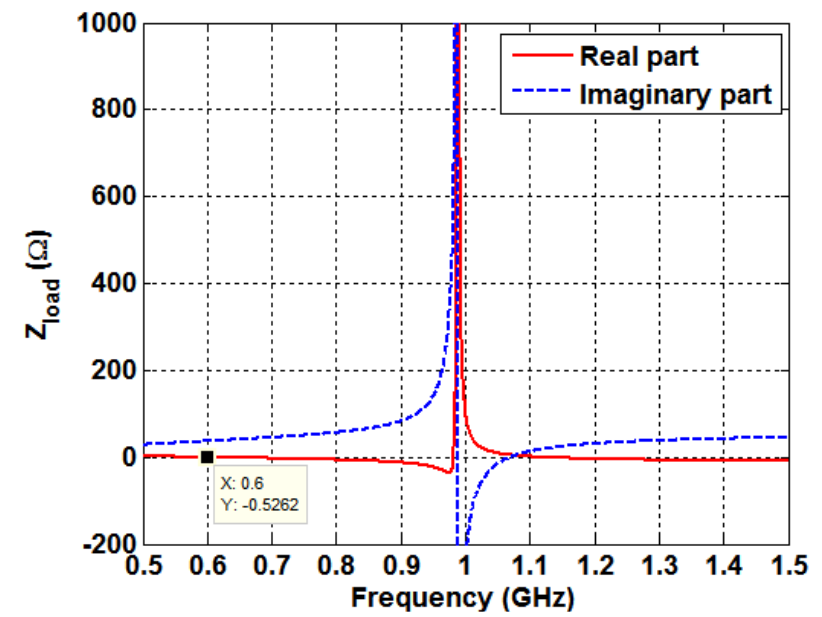

Fig. 7. NIC load impedance to synthetize the needed load for the metasurface.

\section{Improvement of the NIC circuit}

The objective of this section is to improve the NIC circuit so that the load can be achieved with passive components. The proposed solution is based on the addition of a compensation resistor named $R_{\text {comp }}$ at the input of the NIC as shown in Fig. 8. In this condition, the load impedance can be expressed as:

$$
Z_{\text {load,comp }}=-Z_{22}-\frac{Z_{12} \cdot Z_{21}}{Z_{\text {in }}-R_{\text {comp }}-Z_{11}}
$$

Fig. 9 shows the $Z_{\text {load,comp }}$ impedance calculated with $R_{\text {comp }}=50 \Omega$ when $Z_{\text {in }}$ is the impedance of Fig. 3. It can be noticed that the real part of the compensated load impedance $Z_{\text {load,comp }}$ is positive in most of the band of interest. This result is a significant improvement as the load needed is Foster and can now be fabricated with passive components.

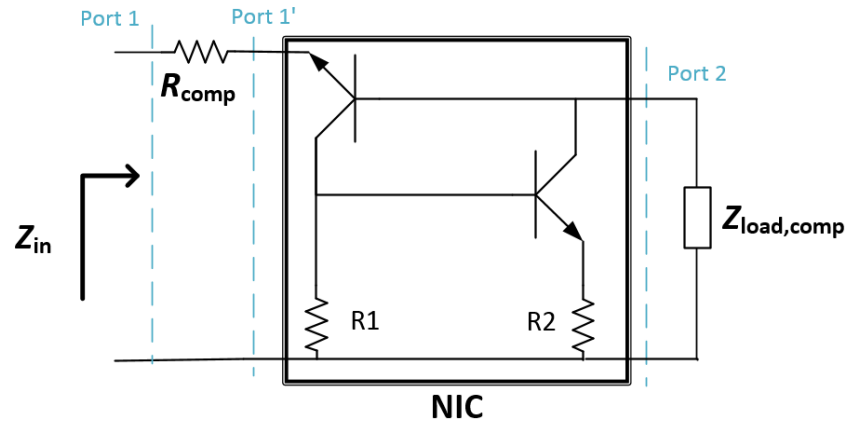

Fig. 8. NIC circuit with the $R_{\text {comp }}$ compensation resistor.

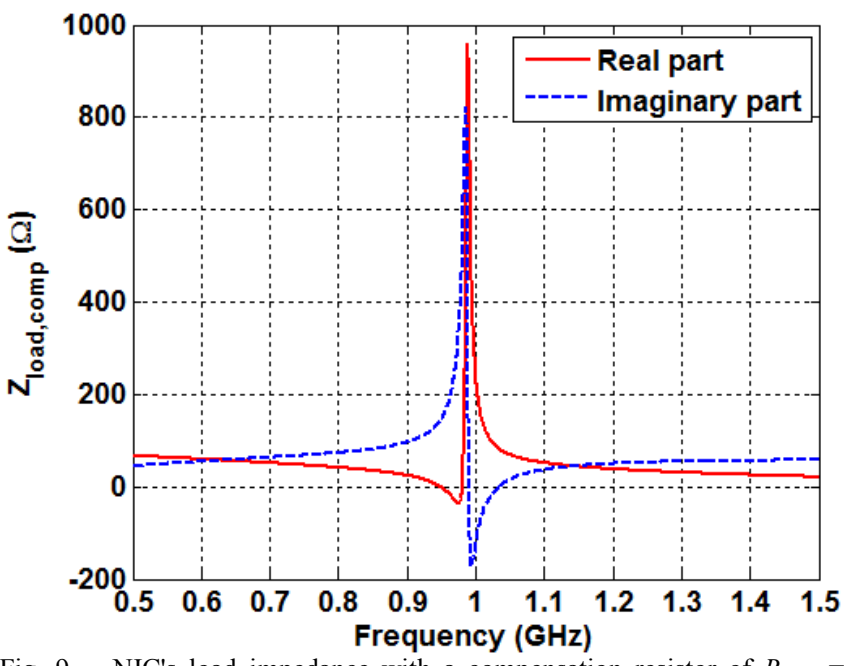

Fig. 9. NIC's load impedance with a compensation resistor of $R_{\text {comp }}=$ $50 \Omega$.

\section{Verification of the optimised NIC circuit and load}

In this section, the reflection coefficient of the active metasurface is computed using the optimized NIC circuit and load. This analysis is carried out using $\mathrm{ADS}^{\mathrm{TM}}$. The results of this simulation are shown in Fig. 10.

Despite a numerical noise, it can be observed that, as expected, the reflection coefficient is equal to 1 in the band of interest. The wideband performance of the active metasurface is therefore validated. 


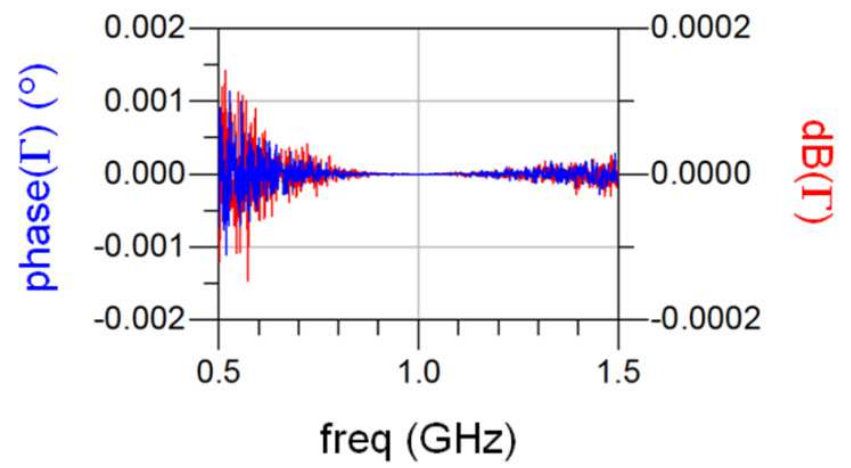

Fig. 10. Reflection coefficient of the active metasurface.

\section{CONCLUSIONS}

In this paper, a procedure has been proposed and implemented to design a wideband active metasurface. The active metasurface includes a passive resonant structure, which is connected at its back to a NIC loaded with passive components.

The NIC has been designed by taking into account the biasing and the effects of the transistors, and by ensuring that the load can be realized with passive components only. The proposed procedure has led to a magnetic surface reflector operating from 0.5 to $1.5 \mathrm{GHz}$. Future work will involve the RLC components determination of the required load and the measurements of the whole structure.

\section{REFERENCES}

[1] D. F. Sievenpiper, "High-Impedance Electromagnetic Surfaces," University of California, Los Angeles, 1999.

[2] F. Gao, F. Zhang, J. Long, M. Jacob, and D. Sievenpiper, "Nondispersive tunable reflection phase shifter based on non-Foster circuits," Electron. Lett., vol. 50, no. 22, pp. 1616-1618, 2014.

[3] J. G. Linvill, "Transistor Negative-Impedance Converters," Proc. IRE, vol. 41, no. 6, pp. 725-729, Jun. 1953.

[4] S. E. Sussman-Fort and R. M. Rudish, "Non-Foster Impedance Matching of Electrically-Small Antennas," IEEE Trans. Antennas Propag., vol. 57, no. 8, pp. 2230-2241, Aug. 2009.

[5] C. Fisné, C. Martel, A. Franc, and N. Raveu, "Metasurface-Based Tunable Reflector for Independent Control of Orthogonal Polarizations," in 2018 12th International Congress on Artificial Materials for Novel Wave Phenomena (Metamaterials), 2018, pp. 125-127.

[6] T. Yanagisawa, "RC Active Networks Using Current Inversion Type Negative Impedance Converters," IRE Trans. Circuit Theory, vol. 4, no. 3, pp. 140-144, Sep. 1957.

[7] S. E. Sussman-Fort, "Gyrator-based biquad filters and negative impedance converters for microwaves," Int. J. RF Microw. Comput.Aided Eng., vol. 8, no. 2, pp. 86-101, Mar. 1998. 